\title{
Where is the brain in all of this?
}

\author{
Vaughan G. Macefield ${ }^{1}$
}

Received: 26 February 2019 / Accepted: 27 February 2019 / Published online: 9 March 2019

(c) Springer-Verlag GmbH Germany, part of Springer Nature 2019

Much of what we have learnt about the organization of the central autonomic network has come from reductionist studies in anaesthetized and reduced animal preparations, with very few in conscious animals. The advent of brain imaging, first with positron emission tomography, but for the last two decades with functional magnetic resonance imaging, has given us unprecedented insight into the operation of the central autonomic network in conscious humans. There is also increasing recognition that, while many diseases featuring autonomic disturbances originate in the peripheral tissues -such as the heart, blood vessels, lungs or kidneys- it is the brain that is ultimately responsible for generating the autonomic responses that aim to maintain the internal environment of the body constant. Of course, these homeostatic adjustments can end up being deleterious, such as the endorgan damage associated with hypertension.

In this issue of Clinical Autonomic Research, Sklerov and colleagues, at the University of North Carolina, have written a very comprehensive review on the state-of-the art of human brain imaging of the central autonomic network [3]. It covers the methodological aspects and, based on a metaanalysis of studies that have used brain imaging to study the human central autonomic network, provides a useful summary of the key areas of the brain involved in cardiovascular control at rest and during maneuvers known to increase muscle sympathetic nerve activity. These studies have shown that the central autonomic network is a distributed network that includes structures in the brainstem, hypothalamus, cingulate cortex, insula, prefrontal cortex and cerebellum.

These are exciting times: in many respects studying the large human brain with functional magnetic resonance imaging is easier than studying the brain of reduced animal preparations and, because research participants can follow instructions, offers the opportunity to study task-related changes in autonomic outflow. Moreover, studying the human brain allows us to study clinical disease states and thereby identify the functional and structural changes in the brain associated with pathophysiology. Indeed, only now, through the use of non-invasive brain imaging, are we starting to understand how the brain brings about, for example, a marked increase in muscle sympathetic nerve activity resulting in neurogenic hypertension in patients with obstructive sleep apnea, a common respiratory disorder during sleep [1, 2]. And this is only one example of endless possibilities. It is exciting that we are starting to fathom where the brain is in all of this.

\section{Compliance with ethical standards}

Conflict of interest The authors declare that there are no conflicts of interest.

\section{References}

1. Henderson LA, Fatouleh RH, Lundblad LC, McKenzie DK, Macefield VG (2016) Effects of 12 months continuous positive airway pressure on sympathetic activity related brainstem function and structure in obstructive sleep apnea. Front Neurosci 10:90

2. Macefield VG, Gandevia SC (1985) Henderson LA (2006) Neural sites involved in the sustained increase in muscle sympathetic nerve activity induced by inspiratory capacity apnea: a fMRI study. J Appl Physiol 100:266-273

3. Sklerov M, Dayan E, Browner N (2018) Functional neuroimaging of the central autonomic network: recent developments and clinical implications. Clin Auton Res. https://doi.org/10.1007/s1028 6-018-0577-0
Vaughan G. Macefield

vaughan.macefield@baker.edu.au

1 Baker Heart and Diabetes Institute, 75 Commercial Rd, Melbourne, Australia 